\title{
A new geometric proof of super-twisting control with actuator saturation $\star$
}

\author{
Abhisek K. Behera ${ }^{\mathrm{a}}$, Asif Chalanga ${ }^{\mathrm{b}}$, Bijnan Bandyopadhyay ${ }^{\mathrm{a}}$ \\ a Interdisciplinary Programme in Systems and Control Engineering, Indian Institute of Technology Bombay, Mumbai-400 076, India \\ ${ }^{\mathrm{b}}$ Laboratoire des Sciences du Numérique de Nantes, Ecole Centrale de Nantes, Nantes 44300, France
}

\begin{abstract}
In this note, the stability of an uncertain system with actuator saturation using super-twisting controller (STC) is analysed. First, a new proof of STC ensuring finite-time stability of the sysyem is proposed using geometric method which gives a new gain conditions. Then, using the proposed proof the domain of attraction (DOA) is explicitly calculated for the system with bounded control.
\end{abstract}

Key words: Super-twisting control; actuator saturation; finite-time stability.

\section{Introduction}

Sliding mode control (SMC) is popularly used for stabilizing the uncertain dynamical systems by a discontinuous control [1]. However, the discontinuous control signal causes wear and tear of the actuator. In the early nineties, a continuous SMC, known as super-twisting control (STC), is proposed that also ensures a sliding mode in finite-time. This control structure is given by

$$
u(t)=-K|s(t)|^{\frac{1}{2}} \operatorname{sign}(s(t))-\int_{0}^{t} L \operatorname{sign}(s(\tau)) \mathrm{d} \tau
$$

that stabilizes an uncertain scalar dynamical system

$$
\dot{s}(t)=a(t)+b(t) u(t)
$$

in finite-time, where $a(t)$ and $b(t)$ are unknown but continuously differentiable scalar functions, and $K$ and $L$ are some positive constants. The control law (1) is studied widely in literature (e.g., [2], [3], [5]-[8], [11]) due to its ability to reject the disturbance completely with continuous control signal. Similarly, the multi-input case is also reported in [4]. Despite of the continuous control, STC may result

\footnotetext{
^ This paper was not presented at any IFAC meeting. Corresponding author Bijnan Bandyopadhyay. Tel. +91 222576 7889. Fax +9122 25700057 .
}

Email addresses: abhisek@sc.iitb.ac.in

(Abhisek K. Behera), asifchalanga@gmail.com

(Asif Chalanga), bijnan@ee.iitb.ac.in

(Bijnan Bandyopadhyay). in a high amplitude oscillations of state trajectory in certain cases [11]. Nevertheless, STC is still considered as an alternative approach to design a continuous SMC. The solutions of the system are absolutely continuous functions that satisfy (2) almost everywhere, and are understood in the Filippov's sense on discontinuous manifold [9].

In this paper, the stability of the system is analysed using STC with actuator saturation which is one of the major concerns in many practical applications. First, a new geometric proof is proposed to show the finite-time stability of STC which is different from the existing ones, e.g., see [2], [5][8]. The main advantage of this proposed proof is that here no difficulty arises for the points on the line $s=0$. The similar proof for super-twisting observer is presented recently in [10] but with a different gain conditions. Then, using the proposed proof the domain of attraction (DOA) is explicitly computed for the actuator saturation such that the system is finite-time stable within this DOA. It is to be noted that the stability of STC with saturating actuator is presented using Lyapunov method in [12]. However, in this paper the stability of STC under actuator saturation is analysed using the proposed geometric proof with an aim of achieving the largest DOA.

The proposed proof follows the idea of constructing system trajectories in the original coordinate instead of in the phase plane. So, the difficulty incurred for the points on the line $s=$ 0 is avoided. Then, using this technique DOA is computed for any given saturation limit. 


\section{Main results}

First, we state some assumptions on the system (2) which hold throughout this paper.

Assumption 1 The function $a$ and its rate are bounded, i.e., $|a|<\bar{a}$ and $|\dot{a}| \leq A$. The function $b$ is bounded and sign definite, i.e., $b \neq 0$, and without loss of generality, $0<\underline{b} \leq b \leq \bar{b}$. Further, we also assume $|\dot{b}| \leq B$.

The STC given in (1) is rewritten as

$$
\begin{aligned}
& u=-K|s|^{\frac{1}{2}} \operatorname{sign}(s)+L_{1} L_{2} v \\
& \dot{v}=-\operatorname{sign}(s)
\end{aligned}
$$

where $L=L_{1} L_{2}$ for some positive constants $L_{1}$ and $L_{2}$. Here, the gains $L_{1}$ and $L_{2}$ allow the flexibility in the design of $L$ as we shall see later. The closed loop system with the control law (3) and (4) is given as

$$
\begin{aligned}
& \dot{s}=b\left(-K|s|^{\frac{1}{2}} \operatorname{sign}(s)+L_{1} \mu\right) \\
& \dot{\mu}=-L_{2} \operatorname{sign}(s)+\frac{\gamma}{L_{1}}
\end{aligned}
$$

where $\mu=L_{2} v+\frac{1}{L_{1}}\left(\frac{a}{b}\right)$ and $\gamma=\frac{\mathrm{d}}{\mathrm{d} t}\left(\frac{a}{b}\right)$. It is easy to see that $|\gamma| \leq \Gamma^{+}$where $\Gamma^{+}=\frac{A \bar{b}+B \bar{a}}{\underline{b}^{2}}$. The classical notions of solution are not applicable since the system (5)-(6) is discontinuous for the points on $s=0$. So, the differential equation on the discontinuous manifold is replaced by an inclusion which is nonempty, closed and bounded, convex and upper semicontinuous in its argument. Then, there exists an absolutely continuous function which satisfies the inclusion almost everywhere, and is regarded as a solution to the system in the Filippov's sense [9].

\subsection{Stability of super-twisting control}

The following Theorem gives the proof of STC without assuming any bound on control which is used later for calculation of DOA.

Theorem 1 Consider the system (5) and (6). Then, the system is finite-time stable if

$$
K>1.8 \sqrt{\frac{L_{1}\left(L_{2}+\frac{\Gamma^{+}}{L_{1}}\right)}{\underline{b}}} \quad \text { and } \quad L_{2}>\frac{\Gamma^{+}}{L_{1}}
$$

where $L_{1}>0$.

PROOF. The proof follows by the construction of geometrical trajectories in each quadrant separately in $(s, \mu)$ plane. Note that every solution of the system (5)-(6) satisfies Filippov's inclusion for all points on the line $s=0$. The system trajectory leaves the line $s=0$ whenever it crosses $s=0$ for nonzero $\mu$ due to (5).

Define the curves $\Sigma_{1} \equiv L_{1} \mu-K|s|^{\frac{1}{2}} \operatorname{sign}(s)=0$ and $\Sigma_{2} \equiv$ $L_{1} \mu+K|s|^{\frac{1}{2}} \operatorname{sign}(s)=0$ as shown in Fig. 1. Clearly, the curve

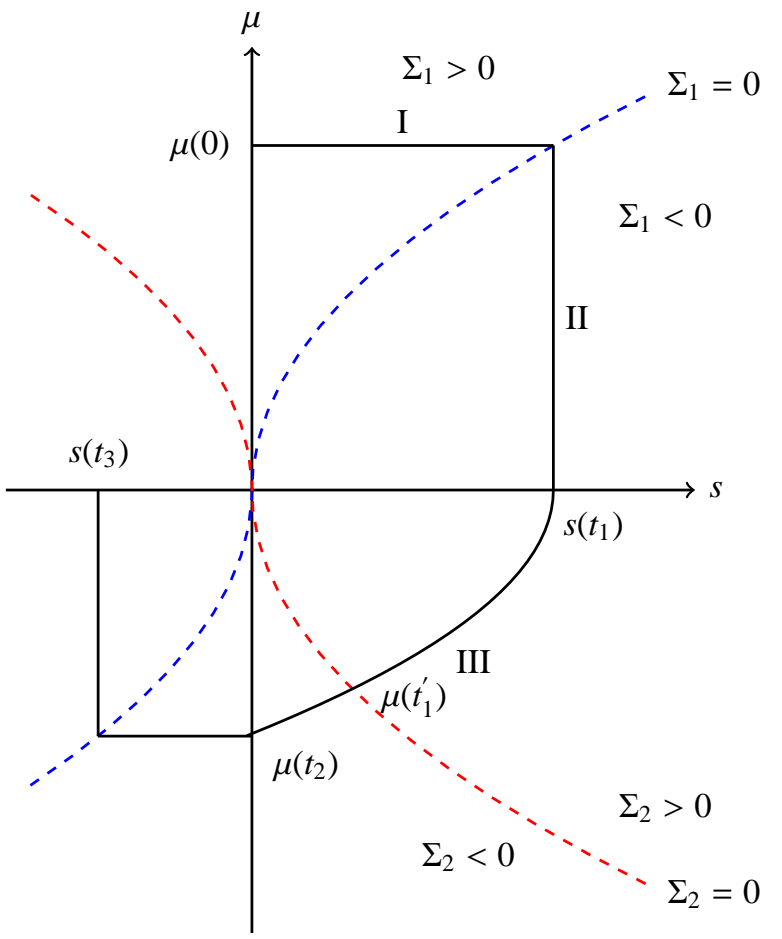

Fig. 1. Majorant curve of STC in $(s, \mu)$ plane.

$\Sigma_{1}=0$ divides the $(s, \mu)$ plane into two parts namely $\Sigma_{1}>0$ and $\Sigma_{1}<0$ such that any trajectory in $\Sigma_{1}>0$ crosses $\Sigma_{1}=0$ before entering $\Sigma_{1}<0$ and vice versa. Similarly for $\Sigma_{2}=0$.

We now proceed to find the trajectory of the system (5) and (6) as shown in Fig. 1. Consider the first quadrant, $s>0$ and $\mu>0$. Any trajectory starting in the region $\Sigma_{1}>0$ with initial condition $(0, \mu(0))$ is bounded by the line segment I due to $L_{2}>\frac{\Gamma^{+}}{L_{1}}$. The equation of segment $\mathrm{I}$ is governed by

$$
\dot{s}>0 \text { and } \dot{\mu}=0 .
$$

So, the line segment starting from the point $(0, \mu(0))$ hits $\Sigma_{1}=0$ at $\left(s\left(t_{1}\right), K\left|s\left(t_{1}\right)\right|^{\frac{1}{2}} / L_{1}\right)$. Then, it enters the region $\Sigma_{1}<0$ in the same quadrant. Similarly, all the trajectories in this region remain bounded by the line segment II which drops from $\left(s\left(t_{1}\right), K\left|s\left(t_{1}\right)\right|^{\frac{1}{2}} / L_{1}\right)$ to $\left(s\left(t_{1}\right), 0\right)$. This is because both $\dot{s}<0$ and $\dot{\mu}<0$ as $\Sigma_{1}<0$ and $L_{2}>\frac{\Gamma^{+}}{L_{1}}$, respectively.

Then, the system trajectory enters into the fourth quadrant ( $s>0$ and $\mu<0$ ) where $\dot{s}<0$ and $\dot{\mu}<0$. It is easy to see that in this quadrant all the trajectories remain bounded by the segment III which is governed by

$$
\begin{aligned}
& \dot{s}=\underline{b} \max \left\{L_{1} \mu,-K|s|^{\frac{1}{2}}\right\} \\
& \dot{\mu}=-\left(L_{2}+\frac{\Gamma^{+}}{L_{1}}\right) .
\end{aligned}
$$

Clearly, the dynamical equations of curve segment III until it reaches the curve $\Sigma_{2}=0$ are represented by $\dot{s}=\underline{b} L_{1} \mu$ and $\dot{\mu}=-\left(L_{2}+\frac{\Gamma^{+}}{L_{1}}\right)$ as $\Sigma_{2}>0$ and $s>0$, respectively. On 
solving these two, the equation of motion of this segment is obtained as

$$
\mu^{2}(t)=\frac{2\left(L_{2}+\frac{\Gamma^{+}}{L_{1}}\right)}{\underline{b} L_{1}}\left(s\left(t_{1}\right)-s(t)\right)
$$

for all $t \in\left[t_{1}, t_{1}^{\prime}\right]$ where $t_{1}^{\prime}$ is the time instant at which the trajectory reaches the curve $\Sigma_{2}=0$. A simple calculation shows that the segment of curve III intersects the curve $\Sigma_{2}=$ 0 at $\left(s\left(t_{1}^{\prime}\right),-K\left|s\left(t_{1}^{\prime}\right)\right|^{\frac{1}{2}} / L_{1}\right)$ where

$$
s\left(t_{1}^{\prime}\right)=\frac{2 L_{1}\left(L_{2}+\frac{\Gamma^{+}}{L_{1}}\right)}{\underline{b} K^{2}+2 L_{1}\left(L_{2}+\frac{\Gamma^{+}}{L_{1}}\right)} s\left(t_{1}\right) .
$$

Once the trajectory reaches $\Sigma_{2}=0$, it moves towards the line $s=0$ due to both $\dot{s}<0$ and $\dot{\mu}<0$. During this, the dynamics of segment III is governed by

$$
\dot{s}=-\underline{b} K|s|^{\frac{1}{2}} \quad \text { and } \quad \dot{\mu}=-\left(L_{2}+\frac{\Gamma^{+}}{L_{1}}\right)
$$

as $\Sigma_{2}<0$. It is seen that with the above dynamical equations the majorant curve now traverses from $\left(s\left(t_{1}^{\prime}\right),-K\left|s\left(t_{1}^{\prime}\right)\right|^{\frac{1}{2}} / L_{1}\right)$ to $\left(0, \mu\left(t_{2}\right)\right)$ and is given as

$$
\mu(t)=\mu\left(t_{1}^{\prime}\right)-\frac{2}{\underline{b} K}\left(L_{2}+\frac{\Gamma^{+}}{L_{1}}\right)\left(\left|s\left(t_{1}^{\prime}\right)\right|^{\frac{1}{2}}-|s(t)|^{\frac{1}{2}}\right) .
$$

This curve hits the line $s=0$ in finite time with the intercept $\mu\left(t_{2}\right)<0$. Using the above relation, we compute the value of this intercept as

$$
\mu\left(t_{2}\right)=-\frac{K}{L_{1}}\left|s\left(t_{1}^{\prime}\right)\right|^{\frac{1}{2}}-\frac{2}{\underline{b} K}\left(L_{2}+\frac{\Gamma^{+}}{L_{1}}\right)\left|s\left(t_{1}^{\prime}\right)\right|^{\frac{1}{2}} .
$$

Using the value of $s\left(t_{1}^{\prime}\right)$ in the above and simplifying further, yields

$$
\frac{\left|\mu\left(t_{2}\right)\right|}{|\mu(0)|}=\frac{\sqrt{2 \underline{b} L_{1}\left(L_{2}+\frac{\Gamma^{+}}{L_{1}}\right) K^{2}+4 L_{1}^{2}\left(L_{2}+\frac{\Gamma^{+}}{L_{1}}\right)^{2}}}{\underline{b}^{2}} .
$$

Denote $\alpha:=\frac{\sqrt{2 \underline{b} L_{1}\left(L_{2}+\frac{\Gamma^{+}}{L_{1}}\right) K^{2}+4 L_{1}^{2}\left(L_{2}+\frac{\Gamma^{+}}{L_{1}}\right)^{2}}}{\underline{b} K^{2}}$. If the right side of (11) less than unity, then the successive intercepts of the system decrease monotonically. So, in order to show $\alpha<1$, it is equivalent to consider that

$$
K^{4}-\frac{2 L_{1}\left(L_{2}+\frac{\Gamma^{+}}{L_{1}}\right)}{\underline{b}} K^{2}-\frac{4 L_{1}^{2}\left(L_{2}+\frac{\Gamma^{+}}{L_{1}}\right)^{2}}{\underline{b}^{2}}>0 .
$$

We consider a quadratic equation in $x=K^{2}$ as $x^{2}+$ $\omega_{1} x+\omega_{2}=0$ with $\omega_{1}=-2 L_{1}\left(L_{2}+\frac{\Gamma^{+}}{L_{1}}\right) / \underline{b}$ and $\omega_{2}=$
$-4 L_{1}^{2}\left(L_{2}+\frac{\Gamma^{+}}{L_{1}}\right)^{2} / \underline{b}^{2}$. Then, it follows immediately that for $x>(1+\sqrt{5}) \frac{L_{1}\left(L_{2}+\frac{\Gamma^{+}}{L_{1}}\right)}{\underline{b}}$, we guarantee $x^{2}+\omega_{1} x+\omega_{2}>0$. So, we conclude if

$$
K>1.8 \sqrt{\frac{L_{1}\left(L_{2}+\frac{\Gamma^{+}}{L_{1}}\right)}{\underline{b}}},
$$

the relation (12) always holds. In other words, any $K$ satisfying (7) yields $0<\alpha<1$. As a consequence, the intercepts by the majorant curve on $\Sigma_{1}=0$ decreases successively in geometric progression. We write the intercepts on the line $s=0$ as $\left\{\left|\mu\left(t_{2 i}\right)\right|\right\}_{i \in \mathbb{Z}_{\geq 0}}=\left\{\alpha^{i}\left|\mu\left(t_{0}\right)\right|\right\}_{i \in \mathbb{Z}_{\geq 0}}$ with $0=t_{0}<t_{2}<\cdots$. So, the system trajectory converges to origin asymptotically.

In order to show finite-time convergence, we find the time of convergence of $\mu$ on the line $s=0$ successively. From (6), we see that for $s>0$,

$$
-\left(L_{2}+\frac{\Gamma^{+}}{L_{1}}\right) \leq \dot{\mu} \leq-\left(L_{2}-\frac{\Gamma^{+}}{L_{1}}\right) .
$$

Solving the right side of differential inequality and using (11), we obtain $t_{2} \leq \frac{1+\alpha}{\left(L_{2}-\frac{\Gamma^{+}}{L_{1}}\right)}|\mu(0)|$. The same time expression may also be obtained for the case $s<0$. Define $T_{i}:=t_{2 i+2}-$ $t_{2 i}$. Then, the total time of convergence is calculated as

$$
\begin{aligned}
T & =\sum_{i \in \mathbb{Z}_{\geq 0}} T_{i} \leq \sum_{i \in \mathbb{Z}_{\geq 0}} \frac{1+\alpha}{\left(L_{2}-\frac{\Gamma^{+}}{L_{1}}\right)} \alpha^{i}|\mu(0)| \\
& =\frac{1+\alpha}{\left(L_{2}-\frac{\Gamma^{+}}{L_{1}}\right)}|\mu(0)| \sum_{i \in \mathbb{Z}_{\geq 0}} \alpha^{i}=\tilde{\alpha} \frac{|\mu(0)|}{\left(L_{2}-\frac{\Gamma^{+}}{L_{1}}\right)}
\end{aligned}
$$

where $\tilde{\alpha}:=\frac{1+\alpha}{1-\alpha}$. In the case $s(0) \neq 0$, the total time of convergence is given as $\tilde{\alpha} \frac{\left|\mu\left(t_{2}\right)\right|}{\left(L_{2}-\frac{\Gamma^{+}}{L_{1}}\right)}+\frac{\left|\mu(0)-\mu\left(t_{2}\right)\right|}{\left(L_{2}-\frac{\Gamma^{+}}{L_{1}}\right)}$. This can be further simplified, using $|\mu(0)| \leq\left|\mu\left(t_{2}\right)\right| / \alpha$, to

$$
T \leq \tilde{\alpha} \frac{\left|\mu\left(t_{2}\right)\right|}{\alpha\left(L_{2}-\frac{\Gamma^{+}}{L_{1}}\right)} .
$$

This shows that the finite-time stability of STC and hence the proof is completed.

One immediate consequence to the Theorem 1 is the following.

Corollary 1 The system, given by (5) and (6), is finite-time stable if the gains satisfy

$$
K>1.8 \sqrt{\frac{L}{\underline{b}}} \text { and } L>\Gamma^{+} .
$$

PROOF. From Theorem 1, the system is finite-time stable if the gains satisfy the relation (7). The gain $L_{1}$ can be 
chosen sufficiently large such that $\frac{\Gamma^{+}}{L_{1}}(=\delta)$ very small and become close to zero as the gain $L_{1}$ is increased further. Similarly, $L_{2}$ can always be designed to ensure $L_{1} L_{2}(=L)$ just greater than $\Gamma^{+}$. So, the gain condition (7) can be written as $K>1.8 \sqrt{\frac{L_{1}\left(L_{2}+\delta\right)}{\underline{b}}}$ and $L>\Gamma^{+}$for any $0<\delta<+\infty$. Now, if the gain $K$ is selected using (14), say $K=\delta_{1} 1.8 \sqrt{\frac{L}{b}}$ for any $\delta_{1}>1$, then there always exists a $\delta>0$ such that $K=\delta_{1} 1.8 \sqrt{\frac{L}{b}}>1.8 \sqrt{\frac{L_{1}\left(L_{2}+\delta\right)}{\underline{b}}}$. This implies that for every gain $K$ satisfying (14), the $L_{1}$ can be selected sufficiently large such that $K$ also satisfies (7). The relation $L>\Gamma^{+}$ follows immediately from (7) by multiplying $L_{1}$ on both the sides of $L_{2}>\frac{\Gamma^{+}}{L_{1}}$. This completes the proof.

Remark 1 For an unperturbed system. i.e., $\dot{s}(t)=u(t)$ with $a(t)=0$ and $b(t)=1$, the gain conditions are found to be $K>0$ and $L>0$ as $L_{1}>0$. Similarly, if $b(t)=1$ but $a(t)$ is a bounded uncertainty then the gains satisfy the relation as $K>1.8 \sqrt{L}$ and $L>\Gamma^{+}$where $\Gamma^{+}=A$.

Remark 2 It may be noted here that the gains $K$ and $L$ do not depend on the controller bound. However, if the uncertain functions become state dependent, the gains of STC depend on the controller bound as the case in [2].

\subsection{Stability with bounded control}

In this section, we present the stability of the system using STC with saturating actuator. Let $U_{M}>0$ be the saturation limit of actuator such that the control is bounded by $U_{M}$, i.e., $|u| \leq U_{M}$. Now, we state the assumption on uncertainty for a given saturation limit.

Assumption $2\left|\frac{a}{b}\right| \leq \frac{\bar{a}}{\underline{b}} \leq q U_{M}$ for some $q \in(0,1)$.

Here, we obtain the largest possible DOA for STC such that the closed loop system is finite-time stable with bounded control within this DOA. Using the results of Theorem 1, we proceed to find this positively invariant region.

Using $\mu=L_{2} v+\frac{1}{L_{1}}\left(\frac{a}{b}\right)$ in (3) gives $\Sigma_{1}=u+\frac{a}{b}$. Since $|u| \leq U_{M}$, it must be ensured $\left|\Sigma_{1}-\frac{a}{b}\right| \leq U_{M}$. Thus, it follows from Assumption 2 that if $\left|\Sigma_{1}\right| \leq(1-q) U_{M}$ then

$$
\begin{aligned}
\left|\Sigma_{1}-\frac{a}{b}\right| & \leq\left|\Sigma_{1}\right|+\frac{\bar{a}}{\underline{b}} \\
& \leq(1-q) U_{M}+q U_{M}=U_{M} .
\end{aligned}
$$

Using these facts, we estimate DOA for STC with bounded control.

Theorem 2 Consider the system (2) subject to bounded control input $|u| \leq U_{M}$. Define

$$
\begin{aligned}
\mathcal{T}_{1}(p):= & \frac{\underline{b} K^{2}+2\left(L+\Gamma^{+}\right)}{\underline{b}}|s|-2(1-q) K U_{M}|s|^{\frac{1}{2}} \\
& +(1-q)^{2} U_{M}^{2}-\frac{2\left(L+\Gamma^{+}\right)}{\underline{b}} p \beta
\end{aligned}
$$

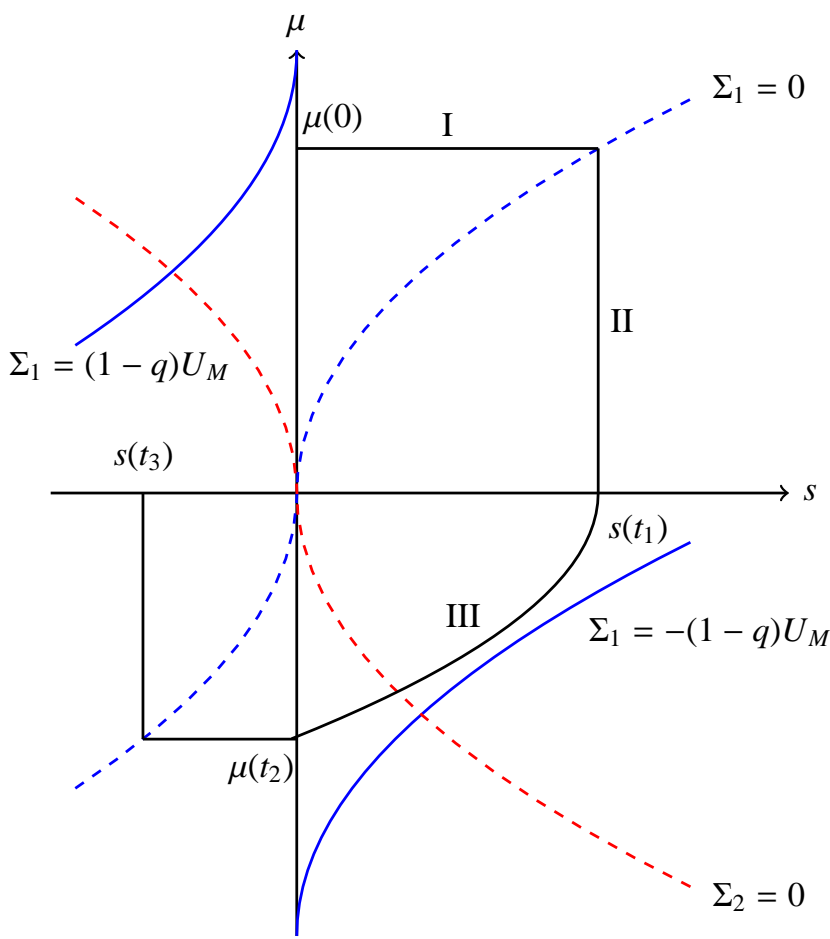

Fig. 2. Domain of attraction of STC with bounded control.

and

$$
\mathcal{T}_{2}(p):=\frac{\underline{b} K^{2}-2\left(L+\Gamma^{+}\right)}{\underline{b} K}|s|^{\frac{1}{2}}-(1-q) U_{M}+\alpha K \sqrt{p \beta}
$$

for any $p \in(0,1)$ and $\beta=\frac{(1-q)^{2} U_{M}^{2}}{K^{2}}$. We denote

$$
p_{1}^{\star}=\underset{p \in(0,1)}{\arg \max } \mathcal{T}_{1}(p)>0 \quad \text { and } \quad p_{2}^{\star}=\underset{p \in(0,1)}{\arg \max } \mathcal{T}_{2}(p)<0
$$

for all $s \in[\lambda p \beta, p \beta]$ and $s \in[0, \lambda p \beta]$, respectively, where $\lambda=\frac{2\left(L+\Gamma^{+}\right)}{\underline{b} K^{2}+2\left(L+\Gamma^{+}\right)}$. Then, if $p^{\star}=\min \left\{p_{1}^{\star}, p_{2}^{\star}\right\}$, the closed loop system (5) and (6) is finite-time stable in DOA given by

$$
\Omega=S \mid \bigcup_{i=1}^{2} \mathcal{S}_{i}
$$

for all $s \in\left[0, p^{\star} \beta\right]$, where

$$
\begin{aligned}
& \mathcal{S}:=\left\{(s, \mu) \in \mathbb{R}^{2}:|s| \leq p^{\star} \beta,|\mu| \leq \frac{K}{L_{1}} \sqrt{p^{\star} \beta}\right\} \\
& \mathcal{S}_{1}:=\left\{(s, \mu) \in \mathbb{R}^{2}:|\mu|-\sqrt{\left.\frac{2\left(L_{2}+\frac{\Gamma^{+}}{L_{1}}\right)}{\underline{b}_{1}}\left(p^{\star} \beta-|s|\right)>0\right\}}\right. \\
& \mathcal{S}_{2}:=\left\{(s, \mu) \in \mathbb{R}^{2}:|\mu|-\frac{K}{L_{1}} \sqrt{\lambda p^{\star} \beta}\right. \\
&\left.-\frac{2}{\underline{b} K}\left(L_{2}+\frac{\Gamma^{+}}{L_{1}}\right)\left(\sqrt{\lambda p^{\star} \beta}-|s|^{\frac{1}{2}}\right)>0\right\} .
\end{aligned}
$$


PROOF. Refer to Fig. 2. Observe that the control signal is less than or equal to saturation limit of the actuator in the region bounded by two curves $\Sigma_{1}=-(1-q) U_{M}$ and $\Sigma_{1}=(1-q) U_{M}$. Clearly, any $\mu(0)$ in the DOA (17) is less than the intercept of the curve $\Sigma_{1}=(1-q) U_{M}$ on the line $s=0$. So, the segments I and II do not contribute towards control saturation. Thus, the idea of the proof follows by constructing the segment III of the trajectory such that it is always constrained by the curve $\Sigma_{1}=-(1-q) U_{M}$, and hence saturation limit is avoided.

First, we find the intercept on the line $\mu=0$ by the curve $\Sigma_{1}+(1-q) U_{M}=0$. We see that it intersects $\mu=0$ at $(\beta, 0)$ where $\beta=\frac{(1-q)^{2} U_{M}^{2}}{K^{2}}$. Let $s\left(t_{1}\right)=p \beta$ for any $p \in(0,1)$. The objective is to find the maximum value of $p$ denoted by $p^{\star}$ such that the segment III does not intersect the curve $\Sigma_{1}+(1-q) U_{M}=0$ for all $s \in\left[0, p^{\star} \beta\right]$.

The equations of segment III are given by (8) and (9). We obtain the condition under which the segment III does not intersect the curve $\Sigma_{1}+(1-q) U_{M}=0$. Using (8), we must ensure

$$
\left|\sqrt{\frac{2\left(L_{2}+\frac{\Gamma^{+}}{L_{1}}\right)}{\underline{b} L_{1}}} \sqrt{s\left(t_{1}\right)-s(t)}\right|<\left.\left|\frac{K}{L_{1}}\right| s(t)\right|^{\frac{1}{2}}-\frac{(1-q)}{L_{1}} U_{M} \mid .
$$

On squaring both sides and then rearranging, it gives $\mathcal{T}_{1}(p)>$ 0 where $\mathcal{T}_{1}(p)$ is given by (15). The maximum value of $p$, denoted by $p_{1}^{\star}$, is calculated such that $\mathcal{T}_{1}\left(p_{1}^{\star}\right)>0$ for all $s \in\left[\lambda p_{1}^{\star} \beta, p_{1}^{\star} \beta\right]$.

Using (9) for the second part of segment III, it must be ensured

$$
\begin{aligned}
& -\left(\frac{\underline{b} K^{2}+2 L_{1}\left(L_{2}+\frac{\Gamma^{+}}{L_{1}}\right)}{\underline{b} K L_{1}}\right)\left|s\left(t_{1}^{\prime}\right)\right|^{\frac{1}{2}} \\
& +\frac{2}{\underline{b} K}\left(L_{2}+\frac{\Gamma^{+}}{L_{1}}\right)|s(t)|^{\frac{1}{2}}>\frac{K}{L_{1}}|s(t)|^{\frac{1}{2}}-\frac{(1-q)}{L_{1}} U_{M} .
\end{aligned}
$$

Rearranging the above relation, gives $\mathcal{T}_{2}(p)<0$. Let $p_{2}^{\star}$ be the maximum value of $p$ such that $\mathcal{T}_{2}\left(p_{2}^{\star}\right)<0$ for all $s \in\left[0, \lambda p_{2}^{\star} \beta\right]$.

To summarize, if $\mathcal{T}_{1}\left(p_{1}^{\star}\right)>0$ and $\mathcal{T}_{2}\left(p_{2}^{\star}\right)<0$, then the segment III remains bounded by $\Sigma_{1}=-(1-q) U_{M}$. Thus, the maximum value of $p$ satisfying both the conditions given by (15) and (16) is given as $p^{\star}=\min \left\{p_{1}^{\star}, p_{2}^{\star}\right\}$.

The same analysis can be carried out for the trajectories in the second quadrant. Now, DOA can be constructed as follows. Note that the sets $\mathcal{S}_{1}$ and $\mathcal{S}_{2}$ denote the regions beyond the curve III in the fourth quadrant, and also beyond the similar curve in the second quadrant. So, DOA can be obtained by excluding these regions from the set $\mathcal{S}$, and is

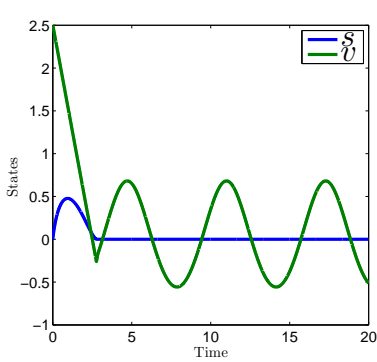

(a) Response of the system.

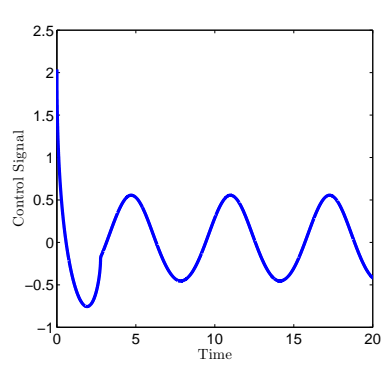

(b) STC within DOA.
Fig. 3. Simulation results of the closed loop system.

given by (17). Hence, the finite-time stability follows from Theorem 1 in DOA given by $\Omega$ in (17). This completes the proof.

Remark 3 The DOA in (17) depends explicitly on the gain $L_{1}$. So, for different values of $L_{1}$, the DOA changes, e.g., large $L_{1}$ reduces DOA and vice versa. So, the largest DOA can be obtained by selecting minimum value of $L_{1}$.

\section{Numerical simulation}

Consider the system (2) with $a(t)=0.5 \sin t$ and $b(t)=$ $1+0.1 \sin t$. Different constants are obtained as $\bar{a}=0.5$, $\underline{b}=0.9, \bar{b}=1.1, A=0.5, B=0.1$. From this $\Gamma^{+}=0.7407$. The gains $L$ and $K$ are chosen using (7) as 0.8148 and 2.3796, respectively. The actuator saturation limit, $U_{M}$, is chosen as 5 and $q=0.1111$. The values of $p_{1}^{\star}$ and $p_{2}^{\star}$ are calculated as 0.62 and 0.659 , respectively. So, $p^{\star}=0.62$. For simulation, the initial condition is chosen as $\left[\begin{array}{ll}0 & 2.5\end{array}\right]^{\top}$ which belongs to DOA given by (17). The simulation is run in MATLAB using Runge-Kutta method of fourth order with a time step of $10^{-3}$. The results are shown in Fig. 3. It is seen that the state $s$ goes to zero in finite-time while the integrator in the control rejects the disturbance as shown in Fig. 3(a). Due to this the control signal becomes equal to the negative of disturbance when the state converges to zero as shown in Fig. 3(b). The control magnitude also remains bounded within the saturation limit in the estimated DOA as proved in Theorem 2 which is also depicted in Fig. 3(b). The plot of system trajectory in $(s, \mu)$ plane is shown in Fig. 4 . The trajectory converges to the origin in finite-time such that it does not intersect with the curve $\Sigma_{1}=-(1-q) U_{M}$. This guarantees that the control signal respects the saturation limit of the actuator.

\section{Conclusion}

In this paper, the finite-time stability of STC is discussed with bounded control magnitude. To analyse the stability, a new geometric proof of STC is proposed in this paper. Then, using this proposed proof, the largest possible DOA is calculated such that the control signal always respects the saturation limit of the actuator while ensuring the finite-time stability of the system which is useful in practice. 


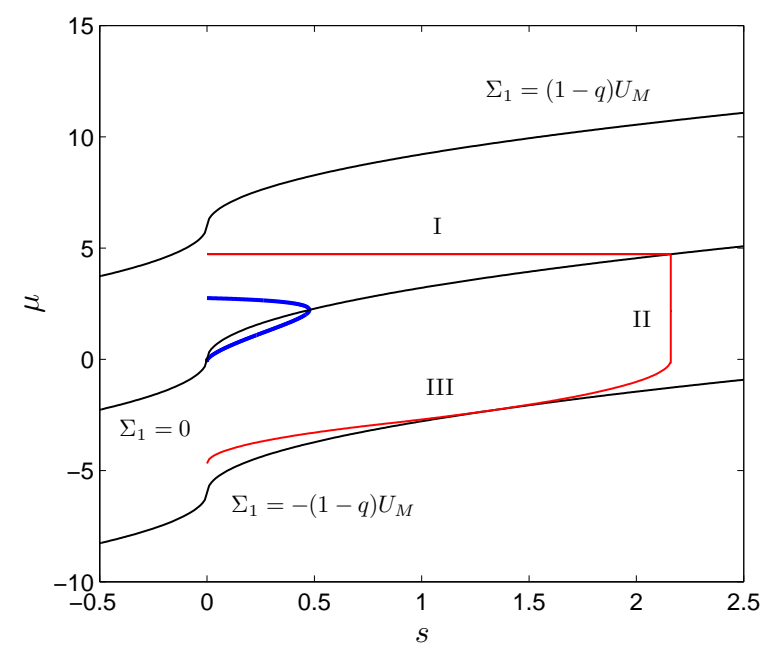

Fig. 4. The system trajectory in $(s, \mu)$ plane.

\section{References}

[1] Utkin, V. (1977). Variable structure systems with sliding modes. IEEE Transactions on Automatic Control, 22(2), 212-222.

[2] Levant, A. (1993). Sliding order and sliding accuracy in sliding mode control. International Journal of Control, 58(6), 1247-1263.

[3] Levant, A. (1998). Robust exact differentiation via sliding mode technique. Automatica, 34(3), 379-384.

[4] Nagesh, I., \& Edwards, C. (2014). A multivariable super-twisting sliding mode approach. Automatica, 50(3), 984-988.

[5] Polyakov, A., \& Poznyak, A. (2009). Reaching time estimation for "super-twisting" second order sliding mode controller via Lyapunov function designing. IEEE Transactions on Automatic Control, 54(8), 1951-1955.

[6] Moreno, J. A., \& Osorio, M. (2012). Strict Lyapunov functions for the super-twisting algorithm. IEEE Transactions on Automatic Control, 57(4), 1035-1040.

[7] Utkin, V. (2013). On convergence time and disturbance rejection of super-twisting control. IEEE Transactions on Automatic Control, 58(8), 2013-2017.

[8] Mu, C., \& Sun, C. (2015). A new finite time convergence condition for super-twisting observer based on Lyapunov analysis. Asian Journal of Control, 17(3), 1-11.

[9] Filippov, A. F. (1988). Differential Equations With Discontinuous Right-Hand Sides, Dordrecht, The Netherlands: Kluwer Academic Publishers.

[10] Kumar, P. R., Behera, A. K., \& Bandyopadhyay, B. (2017). Robust finite-time tracking of Stewart platform: a super-twisting like observer based forward kinematics solution. IEEE Transactions on Industrial Electronics, 64(5), 3776-3785.

[11] Utkin, V. (2016). Discussion aspects of high-order sliding mode control. IEEE Transactions on Automatic Control, 61(3), 829-833.

[12] Castillo, I., Steinberger, M., Fridman, L., Moreno, J. A., \& Horn, M. (2016). Saturated super-twisting algorithm: Lyapunov based approach. International Workshop on Variable Structure Systems, Nanjing, China, 269-273. 